\title{
Bond of Epoxy-Coated Reinforcement: Bar Parameters
}

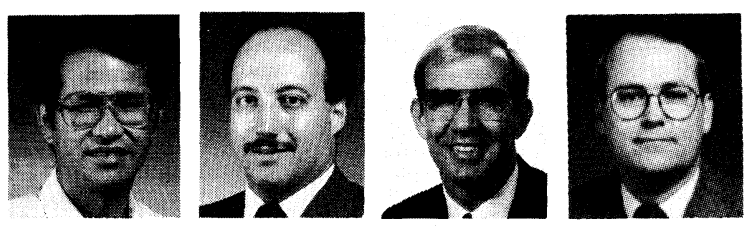

\section{by Oan Chul Choi, Hossain Hadje-Ghaffari, David Darwin, and Steven L. McCabe}

The effects of coating thickness, deformation pattern, and bar size on the reduction in bond strength between reinforcing bars and concrete caused by epoxy coating are described. Tests include beam-end and splice specimens containing No. 5, 6, 8, and 11 bars with average coating thicknesses ranging from 3 to 17 mils $(0.08$ to $0.43 \mathrm{~mm})$. Three deformation patterns are evaluated. All bars are bottom-cast. Beam-end specimens have covers of two bar diameters, while splice specimens have covers that depend on bar size and are less than 2 bar diameters.

The results are compared with the splice tests that were used to establish the epoxy-coated bar provisions in the 1989 ACI Building Code and 1989 AASHTO Bridge Specifications. Epoxy coatings are found to reduce bond strength significantly, but the extent of the reduction is less than that used to select the development length modification factors in the ACI Building Code and AASHTO Bridge Specifications. Coating thickness has little effect on the amount of bond strength reduction for No. 6 bars and larger. However, the thicker the coating, the greater the reduction in bond strength for No. 5 bars. In general, the reduction in bond strength caused by an epoxy coating increases with bar size. The magnitude of the reduction depends on the deformation pattern; bars with relatively larger ribbearing areas with respect to the bar cross section are affected less by the coating than bars with smaller bearing areas. This is the first in a series of papers concerning bond of epoxy-coated reinforcement. Subsequent papers will address the effects of concrete cover, bar position, concrete strength, and transverse reinforcement.

Keywords: bond (concrete to reinforcement); coatings; deformed reinforcement; epoxy resins: lap connections; pullout tests; reinforcing steels; splicing; structural engineering.

Epoxy-coated reinforcing steel has been in general use for about 15 years. Its application to reduce the corrosion of reinforcing steel is increasing each year. While epoxy coating protects the steel, it also reduces the bond between the steel and concrete. The reduction in bond strength has been demonstrated in two principal studies.

Using beam-end specimens containing transverse reinforcement, Johnston and $\mathrm{Zia}^{1}$ observed a 15 percent reduction in bond strength with the use of epoxy-coated bars. Using splices without transverse reinforcement, Treece and $\mathrm{Jirsa}^{2}$ reported an average reduction of 34 percent. Largely based on the recommendations of Treece and Jirsa, ACI Committee $318^{3}$ adopted modification factors to increase the development length for epoxy-coated bars. The factor is 1.5 (a 50 percent increase) for bars with cover less than 3 bar diameters or with clear spacing between bars less than 6 bar diameters. It is 1.2 for all other conditions. AASHTO ${ }^{4}$ has adopted factors of 1.5 and 1.15 based on the same criteria. The new ACI and AASHTO provisions include no recognition of the effect of confining reinforcement on the strength reduction obtained with epoxy coatings.

The 1.5 modification factor is based on only 12 specimens with epoxy-coated reinforcement and 9 specimens with uncoated reinforcement. A single deformation pattern was evaluated, and no specimens were replicated. Considering the high variability typical of bond tests, it is not clear that these few tests provide a reliable picture of the effect of epoxy coating.

This is the first in a series of papers that describe a large-scale study to determine the effect of epoxy coating on bond strength. This paper addresses the effects of parameters associated with the bars themselves: coating thickness, deformation pattern, and bar size. It also considers the effect of embedment length on the relative strength of coated and uncoated bars to establish the suitability of the specimen configurations used in the study. The overall study also considers the effects of concrete cover, bar position, concrete strength, and transverse reinforcement. These topics will be covered in subsequent papers. The full details of this portion of the study are presented in Reference 5.

\section{RESEARCH SIGNIFICANCE}

Epoxy-coated reinforcing bars are used in concrete structures where corrosion protection is a principal design requirement. The bars exhibit a lower bond strength to concrete than uncoated bars. Considering the increasing application of epoxy-coated reinforcement, the conservatism of current design provisions,

ACI Materials Journal, V. 88, No. 2, March-April 1991.

Received Apr. 30, 1990, and reviewed under Institute publication policies. Copyright (ㄷ 1991, American Concrete Institute. All rights reserved, including the making of copies unless permission is obtained from the copyright proprietors. Pertinent discussion will be published in the January-February 1992 ACI Materials Journal if received by Oct. 1, 1991. 
ACI member Oan Chul Choi is a graduate research assistant and PhD candidate in civil engineering at the University of Kansas. He obtained his BS and MS from Seoul National University. He has served as a structural designer at Hyundai Construction Co., Seoul, and taught at Ulsan University in Korea. His research interests include the experimental study of bond and finite element analysis of reinforced concrete.

ACI member Hossain Hadje-Ghaffari is a graduate research assistant and PhD candidate in civil engineering at the University of Kansas. He holds a BS in architectural studies from the University of Nebraska at Lincoln and an MS in civil engineering from the University of Kansas.

David Darwin, FACI, is Deane E. Ackers Professor of Civil Engineering and Director of the Structural Engineering and Materials Laboratory at the University of Kansas. He is a member of the ACI Board of Direction and Technical Activities Committee and is Past President of the Kansas Chapter of ACI. He is also a member and past-chairman of ACI Committee 224, Cracking. He is a member of ACI Committees 408, Bond and Development of Reinforcement, 446, Fracture Mechanics; joint ACI-ASCE Committees 445, Shear and Torsion; 447, Finite Element Analysis of Reinforced Concrete Structures; and the Concrete Materials Research Council.

ACI member Steven L. McCabe is an assistant professor of civil engineering at the University of Kansas. He is active in research involving reinforced concrete, structural analysis/design for dynamic loading, and finite element techniques. McCabe is a member of ACI Committees 439, Steel Reinforcement, and 446, Fracture Mechanics. He also serves on the Board of Direction of the Kansas Chapter of $A C I$.

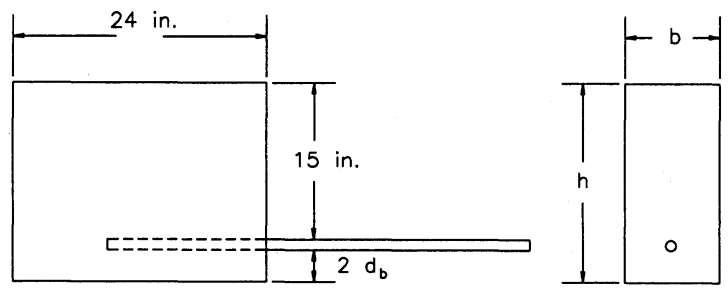

h: 15 in. + bar diameter + cover

b: 9 in. for No.5, No.6 and No.8 bars

10 in. for No.11 bars

(a)

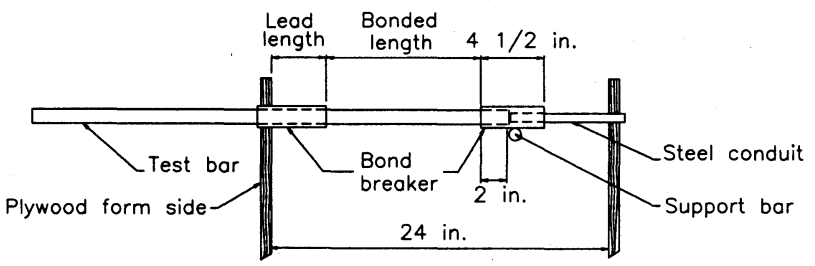

(b)

Fig. 1-(a) Beam-end specimen dimensions; (b) test bar installation $(1 \mathrm{in} .=25.4 \mathrm{~mm})$

and the limited data upon which those provisions are based, an improved understanding of bond behavior is warranted. The goal is to improve economy and constructibility while maintaining an adequate margin of safety.

\section{EXPERIMENTAL PROGRAM}

\section{Test specimens}

Two types of test specimens were tested: beam-end specimens (Fig. 1) and splice specimens (Fig. 2). Beamend specimens containing No. 5, 6, and 8 bars were 9
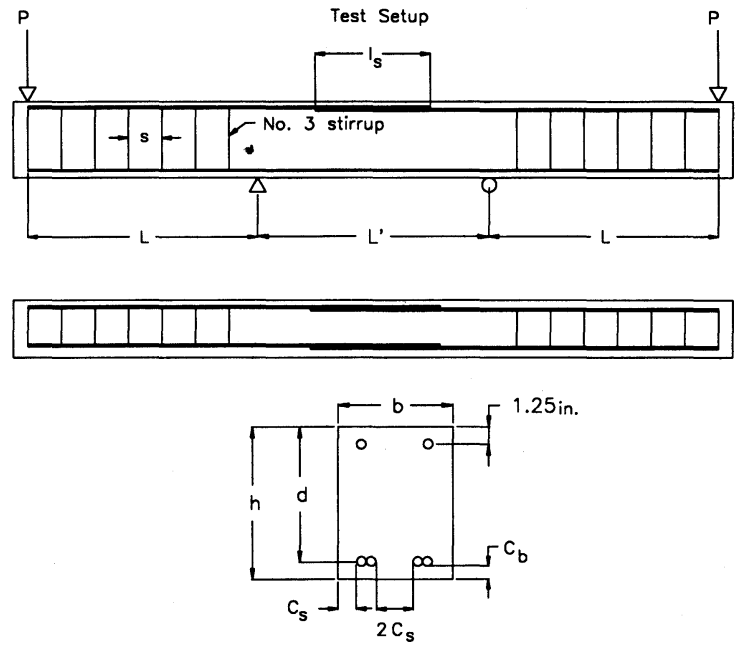

As Cost

\begin{tabular}{|c|c|c|c|c|c|c|c|c|c|c|}
\hline $\begin{array}{c}\text { Bar } \\
\text { No. }\end{array}$ & $\begin{array}{c}L \\
\text { (ft.) }\end{array}$ & $\begin{array}{c}L^{\prime} \\
\text { (ft.) }\end{array}$ & $\begin{array}{c}\text { Is } \\
\text { (in.) }\end{array}$ & $\begin{array}{c}\text { No. of } \\
\text { splices }\end{array}$ & $\begin{array}{c}\text { s } \\
\text { (in.) }\end{array}$ & $\begin{array}{c}b \\
\text { (in.) }\end{array}$ & $\begin{array}{c}d \\
\text { (in.) }\end{array}$ & $\begin{array}{c}\mathrm{h} \\
\text { (in.) }\end{array}$ & $\begin{array}{c}\text { Cs } \\
\text { (in.) }\end{array}$ & $\begin{array}{c}\mathrm{Cb} \\
\text { (in.) }\end{array}$ \\
\hline 5 & 4 & 4 & 12 & 3 & 6 & 15.75 & 14.69 & 16 & 2 & 1 \\
\hline 5 & 4 & 4 & 12 & 2 & 6 & 10.5 & 14.69 & 16 & 2 & 1 \\
\hline 6 & 4 & 4 & 12 & 2 & 7 & 11 & 14.63 & 16 & 2 & 1 \\
\hline 8 & 4 & 4 & 16 & 2 & 7 & 12 & 14 & 16 & 2 & 1.5 \\
\hline 11 & 4.5 & 6 & 24 & 2 & 6 & 13.65 & 13.30 & 16 & 2 & 2 \\
\hline
\end{tabular}

Fig. 2-Splice specimens $(1 \mathrm{in} .=25.4 \mathrm{~mm})$

in. $(229 \mathrm{~mm})$ wide by $24 \mathrm{in} .(610 \mathrm{~mm})$ long. For No. 11 bars, the width was increased to $10 \mathrm{in}$. $(254 \mathrm{~mm})$. Specimen depth was adjusted to provide 15 in. of concrete above the bar and 2 bar diameters of cover below the bar (all bars discussed in this paper were bottom-cast).

Two polyvinyl chloride (PVC) pipes were used as bond breakers to limit the bonded length of the test bar and prevent a cone-type failure on the front face. The bonded lengths of the test bars were selected to insure that the bars did not yield before bond failure occurred. ${ }^{6}$ Standard bonded lengths of $3 \frac{1}{2} 2$ in. $(89 \mathrm{~mm})$ for No. 5 bars, $4 \frac{1 / 2}{2}$ in. $(114 \mathrm{~mm})$ for No. 6 bars, 8 in. $(203 \mathrm{~mm})$ for No. 8 bars, and 9 in. $(229 \mathrm{~mm})$ for No. 11 bars were used. The corresponding lengths of bondbreaking PVC pipe at the front of the bars (lead lengths) were $23 / 8,23 / 4,33 / 4$, and $1 \frac{1}{2}$ in. $(60,70,95$, and $38 \mathrm{~mm}$ ), respectively. Additional specimens were tested to help evaluate the effect of epoxy coating as a function of lead length and bonded length. The results for 270 beam-end specimens are summarized in this paper.

The splice specimens (Fig. 2) consisted of simply supported beams, similar to those tested by Treece and Jirsa. ${ }^{2}$ Splice lengths were 12 in. $(305 \mathrm{~mm})$ for No. 5 and 6 bars, 16 in. $(406 \mathrm{~mm})$ for No. 8 bars, and 24 in. $(610 \mathrm{~mm})$ for No. 11 bars. Each specimen contained two or three splices in the constant moment region. Three splices were used for the No. 5 bars. An additional beam with two splices of uncoated No. 5 bars was used to evaluate the usefulness of double splice specimens for later tests. The strengths of the double and triple splice specimens were nearly proportional to 


\begin{tabular}{|c|c|c|c|c|c|c|c|}
\hline $\begin{array}{l}\text { Bar } \\
\text { size }\end{array}$ & $\begin{array}{c}\text { Deformation } \\
\text { pattern }\end{array}$ & $\begin{array}{c}\text { Yield } \\
\text { strength, } \\
\text { ksi }\end{array}$ & $\begin{array}{l}\text { Deformation } \\
\text { spacing, in. }\end{array}$ & $\begin{array}{c}\text { Deformation } \\
\text { gap, in. }\end{array}$ & $\begin{array}{l}\text { Deformation } \\
\text { angle, deg }\end{array}$ & $\begin{array}{l}\text { Bearing } \\
\text { area } \\
\text { per in.* }\end{array}$ & $\begin{array}{l}\text { Bearing } \\
\text { area } \\
\text { ratio in. }{ }^{-1 *} \\
\end{array}$ \\
\hline $\begin{array}{l}5 \\
5 \\
5\end{array}$ & $\begin{array}{l}\mathrm{S} \\
\mathrm{C} \\
\mathrm{N}\end{array}$ & $\begin{array}{l}70.6 \\
72.3 \\
68.4\end{array}$ & $\begin{array}{l}0.423 \\
0.413 \\
0.379\end{array}$ & $\begin{array}{l}0.159 \\
0.140 \\
0.158\end{array}$ & $\begin{array}{l}90 \\
60 \\
70\end{array}$ & $\begin{array}{l}0.113 \\
0.143 \\
0.166\end{array}$ & $\begin{array}{l}0.361 \\
0.471 \\
0.545\end{array}$ \\
\hline $\begin{array}{l}6 \\
6 \\
6 \\
\end{array}$ & $\begin{array}{l}\mathrm{S} \\
\mathrm{C} \\
\mathrm{N}\end{array}$ & $\begin{array}{l}63.8 \\
70.9 \\
64.2 \\
\end{array}$ & $\begin{array}{l}0.502 \\
0.467 \\
0.462 \\
\end{array}$ & $\begin{array}{l}0.154 \\
0.122 \\
0.151 \\
\end{array}$ & $\begin{array}{l}90 \\
60 \\
70 \\
\end{array}$ & $\begin{array}{l}0.139 \\
0.188 \\
0.201 \\
\end{array}$ & $\begin{array}{l}0.320 \\
0.420 \\
0.448 \\
\end{array}$ \\
\hline $\begin{array}{l}8 \\
8 \\
8 \\
\end{array}$ & $\begin{array}{l}\dot{\mathrm{S}} \\
\mathbf{C} \\
\mathbf{N}\end{array}$ & $\begin{array}{c}67.0 \\
+ \\
63.8\end{array}$ & $\begin{array}{l}0.674 \\
0.656 \\
0.602\end{array}$ & $\begin{array}{l}0.176 \\
0.195 \\
0.160\end{array}$ & $\begin{array}{l}90 \\
60 \\
70\end{array}$ & $\begin{array}{l}0.202 \\
0.241 \\
0.250\end{array}$ & $\begin{array}{l}0.256 \\
0.305 \\
0.316\end{array}$ \\
\hline $\begin{array}{l}11 \\
11 \\
11\end{array}$ & $\begin{array}{l}\mathrm{S} \\
\mathrm{C} \\
\mathrm{N}\end{array}$ & $\begin{array}{l}64.6 \\
63.1 \\
64.3\end{array}$ & $\begin{array}{l}0.945 \\
0.840 \\
0.914\end{array}$ & $\begin{array}{l}0.217 \\
0.196 \\
0.195\end{array}$ & $\begin{array}{l}90 \\
60 \\
70\end{array}$ & $\begin{array}{l}0.313 \\
0.302 \\
0.287\end{array}$ & $\begin{array}{l}0.202 \\
0.196 \\
0.185\end{array}$ \\
\hline
\end{tabular}

*Bearing area based on closely spaced mesurements of ribs; bar areas based on nominal dimensions.

${ }^{+}$Yield strength is greater than $70.0 \mathrm{ksi}$.

1 in. $=25.4 \mathrm{~mm} ; 1 \mathrm{ksi}=6.89 \mathrm{MPa}$, bar sizes: No. $5=16 \mathrm{~mm}$, No. $6=19 \mathrm{~mm}$, No. $8=25 \mathrm{~mm}$, No. $11=35 \mathrm{~mm}$.

the number of splices. Based on this admittedly limited evidence, double splice beams were used for No. 6,8 , and 11 bars. Cover was 1 in. $(25 \mathrm{~mm})$ for No. 5 and 6 bars, $1 \frac{112}{2}$ in. ( $38 \mathrm{~mm}$ ) for No. 8 bars, and 2 in. $(51 \mathrm{~mm})$ for No. 11 bars. The clear spacing between splices was equal to 4 in. $(102 \mathrm{~mm})$ and side cover was equal to 2 in. $(51 \mathrm{~mm})$ for all beams. Additional dimensions and data are included in Fig. 2. The spliced bars were all bottom-cast, in contrast to the Treece/Jirsa specimens, which primarily used top-cast bars. The results for 15 splice specimens are reported in this paper.

\section{Materials}

Reinforcing steel-ASTM A $615^{7}$ Grade 60 , No. 5, 6, 8 , and 11 bars were used. Bars with three deformation patterns, designated $\mathrm{S}, \mathrm{C}$, and N, were tested (Fig. 3). Bars of each size and deformation pattern were from the same heat of steel. Yield strengths and deformation properties are shown in Table 1.

Epoxy coatings were applied in accordance with ASTM A $775^{8}$ and ranged in thickness from 3 to 17 mils $(0.08$ to $0.43 \mathrm{~mm})$ as measured by a pulloff-type thickness gage. ${ }^{8}$ Readings were taken at 6 points around the circumference of the bar between each set of ribs within the bonded length. Average readings within the bonded lengths are reported. A wide range in coating thickness, beyond the ASTM A 775 limits [5 to 12 mils $(0.13$ to $0.30 \mathrm{~mm})$ ], was used to evaluate the effects of coating thickness on bond strength.

Concrete-Nonair-entrained concrete with Type I portland cement and $3 / 4$ in. $(19 \mathrm{~mm})$ nominal maximum size coarse aggregate was used. Water-cement ratios from 0.41 to 0.55 were used to obtain concrete with nominal strengths of 5000 or 6000 psi (34 to $41 \mathrm{MPa}$ ). Concrete of $6000 \mathrm{psi}(41 \mathrm{MPa})$ was used for the majority of the specimens. Mix proportions and concrete properties are listed in Appendix $A^{*}$ and Reference 5. Concrete strengths are listed in Tables 2 and 3.

\footnotetext{
*The appendixes are available in xerographic or similar form from ACI headquarters, where they will be kept permanently on file, at a charge equal to the cost of reproduction plus handling at time of request.
}

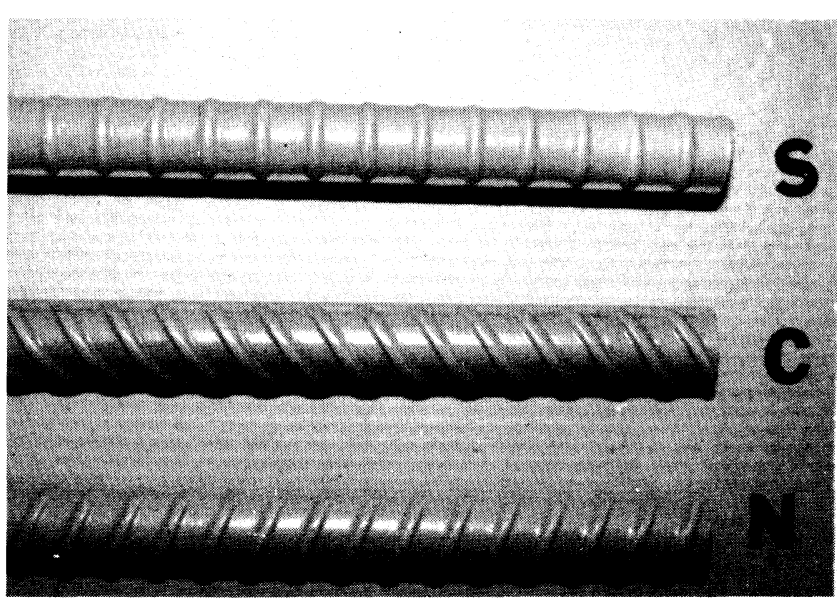

Fig. 3-Reinforcing bar deformation patterns

\section{Test procedure}

The beam-end specimens were tested using apparatus developed by Donahey and Darwin 9 and modified by Brettmann, Darwin, and Donahey. ${ }^{6}$ No. 5 and 6 bars were loaded at approximately $3.0(13.3 \mathrm{kN})$ kips per min. No. 8 and 11 bars were tested at about 6.0 kips $(26.7 \mathrm{kN})$ per min.

Splice specimens were inverted and tested as illustrated in Fig. 2. Splices were located within the constant moment region. Crack locations and widths were recorded during the progress of the tests, which lasted 20 to $25 \mathrm{~min}$.

\section{Results and observations}

Beam-end specimens-Test variables and ultimate bond forces of the individual bars in the beam-end specimens are listed in Appendix B and Reference 5.

Fig. 4 illustrates load-versus-unloaded end slip curves for No. 5 bars. A splitting-type bond failure occurred in all tests. Uncoated bars obtained a higher strength than bars with a nominal 5 mil $(0.13 \mathrm{~mm})$ coating, which in turn had a greater bond strength than bars with a $12 \mathrm{mil}(0.30 \mathrm{~mm})$ coating. The initial slope of the load-slip curve decreases as the coating thickness increases. As will be discussed later, only No. 5 bars exhibited a marked sensitivity to coating thickness. 
Table 2 - Beam splices

\begin{tabular}{|c|c|c|c|c|c|c|c|c|c|c|c|}
\hline Group & $\begin{array}{l}\text { Bar } \\
\text { no. }\end{array}$ & $\begin{array}{c}\text { Deformation } \\
\text { pattern }\end{array}$ & $\begin{array}{c}\text { Splice } \\
\text { length, in. }\end{array}$ & $\begin{array}{c}\text { Average } \\
\text { coating } \\
\text { thickness, mils }\end{array}$ & $\begin{array}{l}\text { Concrete } \\
\text { strength, } \\
\text { psi }\end{array}$ & $\begin{array}{l}\text { No. of } \\
\text { cracks }\end{array}$ & $\begin{array}{c}\text { Widest } \\
\text { crack, mils }\end{array}$ & $\begin{array}{l}\text { Bar stress } \\
\text { for crack } \\
\text { comparison, ksi }\end{array}$ & $\begin{array}{c}\text { Ultimate } \\
\text { moment, k-in. }\end{array}$ & $\begin{array}{l}\text { Ultimate } \\
\text { stress, ksi }\end{array}$ & $C / U^{*}$ \\
\hline SP1 & $\begin{array}{r}5 \\
5^{\dagger} \\
5^{\dagger}\end{array}$ & $\begin{array}{l}\mathbf{N} \\
\mathbf{N} \\
\mathbf{N}\end{array}$ & $\begin{array}{l}12 \\
12 \\
12\end{array}$ & $\begin{array}{l}0.0 \\
0.0 \\
9.5\end{array}$ & 5360 & $\begin{array}{l}7 \\
8 \\
6\end{array}$ & $\begin{array}{l}9 \\
7 \\
7\end{array}$ & $\begin{array}{r}40.9 \\
42.1 \\
42.1\end{array}$ & $\begin{array}{l}521 \\
813 \\
609\end{array}$ & $\begin{array}{l}58.7 \\
61.2 \\
45.5\end{array}$ & 0.74 \\
\hline SP2 & $\begin{array}{l}6 \\
6 \\
6 \\
6\end{array}$ & $\begin{array}{l}S \\
S \\
C \\
C\end{array}$ & $\begin{array}{l}12 \\
12 \\
12 \\
12\end{array}$ & $\begin{array}{l}0.0 \\
8.3 \\
0.0 \\
8.8\end{array}$ & 6010 & $\begin{array}{l}6 \\
3 \\
5 \\
6\end{array}$ & $\begin{array}{l}7 \\
9 \\
5 \\
5\end{array}$ & $\begin{array}{l}36.7 \\
36.7 \\
36.7 \\
36.7\end{array}$ & $\begin{array}{l}543 \\
511 \\
610 \\
466\end{array}$ & $\begin{array}{l}43.2 \\
40.6 \\
48.7 \\
36.9\end{array}$ & $\begin{array}{l}0.94 \\
0.76\end{array}$ \\
\hline SP3 & $\begin{array}{l}8 \\
8 \\
8 \\
8\end{array}$ & $\begin{array}{l}S \\
S \\
\mathbf{N} \\
\mathbf{N}\end{array}$ & $\begin{array}{l}16 \\
16 \\
16 \\
16\end{array}$ & $\begin{array}{l}0.0 \\
9.4 \\
0.0 \\
9.5\end{array}$ & 5980 & $\begin{array}{l}6 \\
4 \\
5 \\
7\end{array}$ & $\begin{array}{l}7 \\
5 \\
9 \\
7\end{array}$ & $\begin{array}{l}25.9 \\
25.9 \\
25.9 \\
25.9\end{array}$ & $\begin{array}{l}854 \\
768 \\
858 \\
737\end{array}$ & $\begin{array}{l}40.1 \\
35.9 \\
40.3 \\
34.4\end{array}$ & $\begin{array}{l}0.90 \\
0.85\end{array}$ \\
\hline SP4 & $\begin{array}{l}11 \\
11 \\
11 \\
11\end{array}$ & $\begin{array}{l}\mathrm{S} \\
\mathrm{S} \\
\mathrm{C} \\
\mathrm{C}\end{array}$ & $\begin{array}{l}24 \\
24 \\
24 \\
24\end{array}$ & $\begin{array}{r}0.0 \\
9.3 \\
0.0 \\
10.3\end{array}$ & 5850 & $\begin{array}{l}5 \\
5 \\
7 \\
6\end{array}$ & $\begin{array}{r}7 \\
9 \\
7 \\
10\end{array}$ & $\begin{array}{l}24.0 \\
24.0 \\
24.0 \\
24.0\end{array}$ & $\begin{array}{l}1459 \\
1053 \\
1372 \\
1128\end{array}$ & $\begin{array}{l}37.6 \\
26.6 \\
35.2 \\
28.6\end{array}$ & $\begin{array}{l}0.71 \\
0.81\end{array}$ \\
\hline
\end{tabular}

${ }^{*} C / U=$ ratio of bond strengths of coated to uncoated bars.

${ }^{\dagger}$ These beams contained 3 splices.

1 in. $=25.4 \mathrm{~mm} ; 1 \mathrm{mil}=0.001 \mathrm{in} .=0.025 \mathrm{~mm} ; 1 \mathrm{psi}=6.89 \mathrm{kPa} ; 1 \mathrm{ksi}=6.89 \mathrm{MPa} ; 1 \mathrm{k}-\mathrm{in} .=0.113 \mathrm{~N}-\mathrm{m}$

Table 3(a) - Summary of beam-end tests for specimens with standard configuration

\begin{tabular}{|c|c|c|c|c|c|c|c|c|c|c|}
\hline $\begin{array}{l}\text { Bar } \\
\text { size }\end{array}$ & $\begin{array}{c}\text { Deformation } \\
\text { pattern }\end{array}$ & $\begin{array}{c}\text { Group } \\
\text { no. }\end{array}$ & $\begin{array}{l}\text { Concrete } \\
\text { strength, } \\
\text { psi }\end{array}$ & $\begin{array}{c}\text { No. of } \\
\text { uncoated } \\
\text { bars }\end{array}$ & $\begin{array}{c}\text { Uncoated } \\
\text { bars } \\
\text { bond } \\
\text { force, } \mathrm{lb}\end{array}$ & $\begin{array}{l}\text { No. of } \\
\text { coated } \\
\text { bars }\end{array}$ & $\begin{array}{c}\text { Coated } \\
\text { bars } \\
\text { bond } \\
\text { force, } \mathrm{lb}\end{array}$ & $\begin{array}{l}C / U^{\dagger} \\
\text { group }\end{array}$ & $\begin{array}{l}U / U,+ \\
\text { all }\end{array}$ & $\begin{array}{c}C / U,{ }^{\dagger} \\
\text { all }\end{array}$ \\
\hline $\begin{array}{l}5 \\
5 \\
\end{array}$ & $\begin{array}{l}\mathrm{S} \\
\mathrm{S}\end{array}$ & $\begin{array}{r}9 \\
21 \\
\end{array}$ & $\begin{array}{l}5650 \\
5990 \\
\end{array}$ & $\begin{array}{l}3 \\
3 \\
\end{array}$ & $\begin{array}{l}14,154 \\
14,598 \\
\end{array}$ & $\begin{array}{l}6 \\
6 \\
\end{array}$ & $\begin{array}{l}11,753 \\
12,005 \\
\end{array}$ & $\begin{array}{l}0.83 \\
0.82 \\
\end{array}$ & $\begin{array}{l}1.01 \\
104 \\
\end{array}$ & $\begin{array}{l}0.84 \\
0.86 \\
\end{array}$ \\
\hline \multicolumn{5}{|c|}{ Average $=$} & \multicolumn{2}{|l|}{14,376} & \multicolumn{2}{|l|}{11,879} & 1.03 & 0.85 \\
\hline $\begin{array}{l}5 \\
5 \\
\end{array}$ & $\begin{array}{l}\mathrm{C} \\
\mathrm{C}\end{array}$ & $\begin{array}{l}10 \\
21\end{array}$ & $\begin{array}{l}5990 \\
5990 \\
\end{array}$ & $\begin{array}{l}3 \\
3 \\
\end{array}$ & $\begin{array}{l}13,580 \\
15,078 \\
\end{array}$ & $\begin{array}{l}6 \\
6 \\
\end{array}$ & $\begin{array}{l}13,009 \\
13,020 \\
\end{array}$ & $\begin{array}{l}0.96 \\
0.86\end{array}$ & $\begin{array}{l}0.97 \\
1.08 \\
\end{array}$ & $\begin{array}{l}0.93 \\
0.93\end{array}$ \\
\hline \multicolumn{4}{|c|}{ Average $=$} & \multicolumn{3}{|c|}{14,329} & 13,014 & 0.91 & 1.02 & 0.93 \\
\hline $\begin{array}{l}5 \\
5 \\
5 \\
\end{array}$ & $\begin{array}{l}\mathbf{N} \\
\mathbf{N} \\
\mathbf{N}\end{array}$ & $\begin{array}{l}11 \\
12 \\
13 \\
\end{array}$ & $\begin{array}{l}5970 \\
5940 \\
5840 \\
\end{array}$ & $\begin{array}{l}3 \\
3 \\
3 \\
\end{array}$ & $\begin{array}{l}12,964 \\
14,003 \\
13,107 \\
\end{array}$ & $\begin{array}{l}3 \\
3 \\
3 \\
\end{array}$ & $\begin{array}{l}11,998 \\
12,425 \\
11,977 \\
\end{array}$ & $\begin{array}{l}0.93 \\
0.89 \\
0.91 \\
\end{array}$ & $\begin{array}{l}0.92 \\
1.00 \\
0.93 \\
\end{array}$ & $\begin{array}{l}0.86 \\
0.89 \\
0.85 \\
\end{array}$ \\
\hline \multicolumn{4}{|c|}{ Average $=$} & \multicolumn{3}{|c|}{13,358} & 12,133 & 0.91 & 0.95 & 0.87 \\
\hline \multicolumn{4}{|c|}{ Average of all No. 5 bars $^{\S}=$} & \multicolumn{3}{|c|}{14,021} & 12,342 & 0.88 & 1.00 & 0.88 \\
\hline
\end{tabular}

*Numerator and denominator based on group average.

${ }^{\dagger}$ Numerator based on group average. Denominator based on average for three deformation patterns for each bar size; each deformation pattern weighted equally.

${ }^{8}$ Each deformation pattern weighted equally.

$1 \mathrm{psi}=6.89 \mathrm{kPa} ; 1 \mathrm{lb}=4.45 \mathrm{~N}$.



Fig. 4-Load-slip curves for S-pattern No. 5 bars (1 lb $=4.45 \mathrm{~N} ; 1 \mathrm{in} .=25.4 \mathrm{~mm}$ )

Splice specimens-The load-deflection curves (applied load-versus-center line deflection minus average load-point deflection) for the splice specimens (Fig. 5) indicate little difference in the response of the members, with the principal exception that epoxy-coated bar

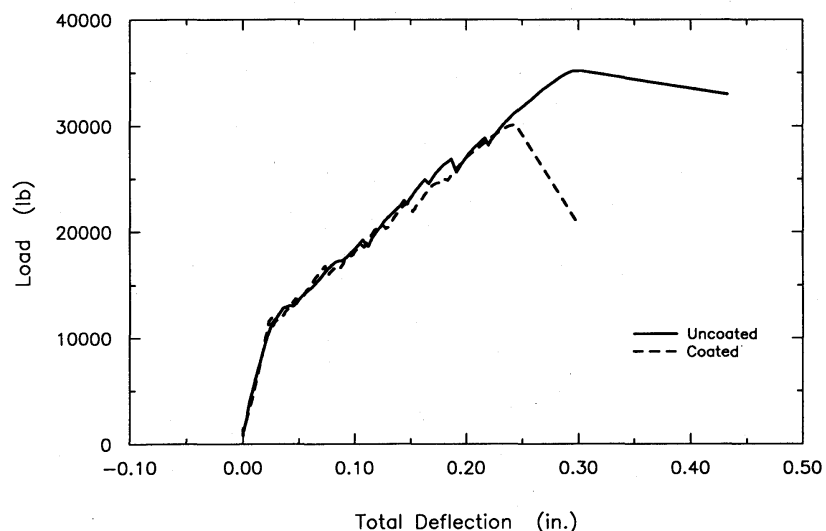

Fig. 5-Load-deflection curves for S-pattern No. 8 bar splice specimens $(1 \mathrm{lb}=4.45 \mathrm{~N} ; 1 \mathrm{in} .=25.4 \mathrm{~mm})$

specimens consistently failed at a lower load than uncoated bar specimens.

Crack widths were measured within a region spanning $12 \mathrm{in.}(305 \mathrm{~mm})$ on either side of the splice. The number of cracks and maximum crack widths are sum- 
Table 3(b) - Summary of beam-end tests for specimens with standard configuration

\begin{tabular}{|c|c|c|c|c|c|c|c|c|c|c|}
\hline $\begin{array}{l}\text { Bar } \\
\text { size }\end{array}$ & $\begin{array}{c}\text { Deformation } \\
\text { pattern }\end{array}$ & $\begin{array}{c}\text { Group } \\
\text { no. }\end{array}$ & $\begin{array}{c}\text { Concrete } \\
\text { strength, } \\
\text { psi }\end{array}$ & $\begin{array}{c}\text { No. of } \\
\text { uncoated } \\
\text { bars }\end{array}$ & $\begin{array}{c}\text { Uncoated } \\
\text { bars } \\
\text { bond } \\
\text { force, } \\
\mathrm{lb}\end{array}$ & $\begin{array}{l}\text { No. of } \\
\text { coated } \\
\text { bars }\end{array}$ & $\begin{array}{c}\text { Coated } \\
\text { bars } \\
\text { bond } \\
\text { force, } \\
\mathrm{lb}\end{array}$ & $\begin{array}{l}C / U^{*} \\
\text { group }\end{array}$ & $\begin{array}{c}U / U,{ }^{\dagger} \\
\text { all }\end{array}$ & $\begin{array}{c}C / U,{ }^{\dagger} \\
\quad \text { all }\end{array}$ \\
\hline $\begin{array}{l}6 \\
6 \\
\end{array}$ & $\begin{array}{l}S \\
S \\
\end{array}$ & $\begin{array}{l}14 \\
17 \\
\end{array}$ & $\begin{array}{l}5800 \\
5850 \\
\end{array}$ & $\begin{array}{l}3 \\
3 \\
\end{array}$ & $\begin{array}{l}19,363 \\
18,720 \\
\end{array}$ & $\begin{array}{l}6 \\
6 \\
\end{array}$ & $\begin{array}{l}15,498 \\
15,525 \\
\end{array}$ & $\begin{array}{l}0.80 \\
0.83 \\
\end{array}$ & $\begin{array}{l}1.00 \\
0.97 \\
\end{array}$ & $\begin{array}{l}0.80 \\
0.81 \\
\end{array}$ \\
\hline \multicolumn{5}{|c|}{ Average $=$} & \multicolumn{2}{|l|}{19,041} & \multicolumn{2}{|l|}{15,511} & 0.99 & 0.80 \\
\hline $\begin{array}{l}6 \\
6 \\
\end{array}$ & $\begin{array}{l}\mathrm{C} \\
\mathrm{C}\end{array}$ & $\begin{array}{l}14 \\
17 \\
\end{array}$ & $\begin{array}{l}5800 \\
5850 \\
\end{array}$ & $\begin{array}{l}3 \\
3 \\
\end{array}$ & $\begin{array}{l}18,733 \\
18,760 \\
\end{array}$ & $\begin{array}{l}6 \\
6 \\
\end{array}$ & $\begin{array}{l}18,112 \\
16,056 \\
\end{array}$ & $\begin{array}{l}0.97 \\
0.86 \\
\end{array}$ & $\begin{array}{l}0.97 \\
0.97 \\
\end{array}$ & $\begin{array}{l}0.94 \\
0.83 \\
\end{array}$ \\
\hline \multicolumn{4}{|c|}{ Average $=$} & \multicolumn{3}{|c|}{18,746} & 17,084 & 0.91 & 0.97 & 0.89 \\
\hline $\begin{array}{l}6 \\
6 \\
\end{array}$ & $\begin{array}{l}\mathbf{N} \\
\mathbf{N} \\
\end{array}$ & $\begin{array}{l}14 \\
22 \\
\end{array}$ & $\begin{array}{l}5800 \\
6300 \\
\end{array}$ & $\begin{array}{l}3 \\
6 \\
\end{array}$ & $\begin{array}{l}19,309 \\
20,385 \\
\end{array}$ & $\begin{array}{l}3 \\
6 \\
\end{array}$ & $\begin{array}{l}19,089 \\
18,486 \\
\end{array}$ & $\begin{array}{l}0.99 \\
0.91 \\
\end{array}$ & $\begin{array}{l}1.00 \\
1.06 \\
\end{array}$ & $\begin{array}{l}0.99 \\
0.96 \\
\end{array}$ \\
\hline \multicolumn{4}{|c|}{ Average $=$} & \multicolumn{3}{|c|}{20,026} & 18,687 & 0.93 & 1.04 & 0.97 \\
\hline \multicolumn{4}{|c|}{ Average of all No. 6 bars $^{\S}$} & & 19,271 & & 17,094 & 0.89 & 1.00 & 0.89 \\
\hline
\end{tabular}

*Numerator and denominator based on group average.

${ }^{\dagger}$ Numerator based on group average. Denominator based on average for three deformation patterns for each bar size; each deformation pattern weighted equally.

${ }^{8}$ Each deformation pattern weighted equally.

$1 \mathrm{psi}=6.89 \mathrm{kPa} ; 1 \mathrm{lb}=4.45 \mathrm{~N}$.

Table 3(c) - Summary of beam-end tests for specimens with standard configuration

\begin{tabular}{|c|c|c|c|c|c|c|c|c|c|c|}
\hline $\begin{array}{l}\text { Bar } \\
\text { size }\end{array}$ & $\begin{array}{l}\text { Deformation } \\
\text { pattern }\end{array}$ & $\begin{array}{c}\text { Group } \\
\text { no. }\end{array}$ & $\begin{array}{c}\text { Concrete } \\
\text { strength, } \\
\text { psi }\end{array}$ & $\begin{array}{c}\text { No. of } \\
\text { uncoated } \\
\text { bars }\end{array}$ & $\begin{array}{c}\text { Uncoated } \\
\text { bars } \\
\text { bond } \\
\text { force, } \\
\text { lb }\end{array}$ & $\begin{array}{l}\text { No. of } \\
\text { coated } \\
\text { bars }\end{array}$ & $\begin{array}{c}\text { Coated } \\
\text { bars } \\
\text { bond } \\
\text { force, } \\
\mathrm{lb}\end{array}$ & $\begin{array}{l}C / U^{*} \\
\text { group }\end{array}$ & $\begin{array}{c}U / U,{ }^{\dagger} \\
\quad \text { all }\end{array}$ & $\begin{array}{c}C / U,{ }^{\dagger} \\
\text { all }\end{array}$ \\
\hline $\begin{array}{l}8 \\
8 \\
8 \\
8\end{array}$ & $\begin{array}{l}S \\
S \\
S \\
S\end{array}$ & $\begin{array}{r}3 \\
6 \\
15 \\
18\end{array}$ & $\begin{array}{c}6090 \\
5870 \\
6000 \\
4790-5430\end{array}$ & $\begin{array}{l}3 \\
2 \\
2 \\
3\end{array}$ & $\begin{array}{l}41,384 \\
45,104 \\
42,680 \\
41,312\end{array}$ & $\begin{array}{l}9 \\
2 \\
6 \\
3\end{array}$ & $\begin{array}{l}29,472 \\
34,512 \\
31,600 \\
34,064\end{array}$ & $\begin{array}{l}0.71 \\
0.77 \\
0.74 \\
0.82\end{array}$ & $\begin{array}{l}0.96 \\
1.05 \\
0.99 \\
0.96\end{array}$ & $\begin{array}{l}0.68 \\
0.80 \\
0.73 \\
0.79\end{array}$ \\
\hline \multicolumn{4}{|c|}{ Average $=$} & \multicolumn{3}{|c|}{42,365} & 31,303 & 0.74 & 0.98 & 0.73 \\
\hline $\begin{array}{l}8 \\
8 \\
8 \\
\end{array}$ & $\begin{array}{l}\mathrm{C} \\
\mathrm{C} \\
\mathrm{C}\end{array}$ & $\begin{array}{l}2 \\
5 \\
6 \\
\end{array}$ & $\begin{array}{l}5700 \\
5920 \\
5870 \\
\end{array}$ & $\begin{array}{l}1 \\
3 \\
2 \\
\end{array}$ & $\begin{array}{l}47,184 \\
36,504 \\
45,880\end{array}$ & $\begin{array}{l}3 \\
9 \\
2 \\
\end{array}$ & $\begin{array}{l}37,976 \\
34,784 \\
35,600\end{array}$ & $\begin{array}{l}0.80 \\
0.95 \\
0.78\end{array}$ & $\begin{array}{l}1.10 \\
0.85 \\
1.07\end{array}$ & $\begin{array}{l}0.88 \\
0.81 \\
0.83\end{array}$ \\
\hline \multicolumn{4}{|c|}{ Average $=$} & \multicolumn{3}{|c|}{41,409} & 35,584 & 0.90 & 0.96 & 0.83 \\
\hline $\begin{array}{l}8 \\
8 \\
8 \\
8\end{array}$ & $\begin{array}{l}\mathbf{N} \\
\mathbf{N} \\
\mathbf{N} \\
\mathbf{N}\end{array}$ & $\begin{array}{r}4 \\
6 \\
15 \\
18 \\
\end{array}$ & $\begin{array}{c}6130 \\
5870 \\
6000 \\
4790-5430 \\
\end{array}$ & $\begin{array}{l}3 \\
2 \\
3 \\
3 \\
\end{array}$ & $\begin{array}{l}46,104 \\
43,304 \\
43,464 \\
48,256 \\
\end{array}$ & $\begin{array}{l}3 \\
2 \\
0 \\
3 \\
\end{array}$ & $\begin{array}{r}37,208 \\
41,296 \\
0 \\
38,800 \\
\end{array}$ & $\begin{array}{l}0.81 \\
0.95 \\
0.00 \\
0.80 \\
\end{array}$ & $\begin{array}{l}1.07 \\
1.01 \\
1.01 \\
1.12 \\
\end{array}$ & $\begin{array}{l}0.86 \\
0.96 \\
0.00 \\
0.90\end{array}$ \\
\hline \multicolumn{4}{|c|}{ Average $=$} & \multicolumn{3}{|c|}{45,461} & 38,827 & 0.84 & 1.06 & 0.90 \\
\hline \multicolumn{4}{|c|}{ Average of all No. 8 bars $^{\S}=$} & & 43,078 & & 35,238 & 0.83 & 1.00 & 0.82 \\
\hline
\end{tabular}

Table 3(d) - Summary of beam-end tests for specimens with standard configuration

\begin{tabular}{|c|c|c|c|c|c|c|c|c|c|c|}
\hline $\begin{array}{l}\text { Bar } \\
\text { size }\end{array}$ & $\begin{array}{c}\text { Deformation } \\
\text { pattern }\end{array}$ & $\begin{array}{c}\text { Group } \\
\text { no. }\end{array}$ & $\begin{array}{c}\text { Concrete } \\
\text { strength, } \\
\text { psi }\end{array}$ & $\begin{array}{c}\text { No. of } \\
\text { uncoated } \\
\text { bars }\end{array}$ & $\begin{array}{c}\text { Uncoated } \\
\text { bars } \\
\text { bond } \\
\text { force, } \\
\text { lb }\end{array}$ & $\begin{array}{l}\text { No. of } \\
\text { coated } \\
\text { bars }\end{array}$ & $\begin{array}{c}\text { Coated } \\
\text { bars } \\
\text { bond } \\
\text { force, } \\
\text { lb }\end{array}$ & $\begin{array}{l}C / U^{*} \\
\text { group }\end{array}$ & $\begin{array}{c}U / U,{ }^{\dagger} \\
\text { all }\end{array}$ & $\begin{array}{c}C / U,{ }^{\dagger} \\
\text { all }\end{array}$ \\
\hline $\begin{array}{l}11 \\
11 \\
\end{array}$ & $\begin{array}{l}\mathrm{S} \\
\mathrm{S}\end{array}$ & $\begin{array}{l}19 \\
20 \\
\end{array}$ & $\begin{array}{l}5070-5270 \\
5260-5290 \\
\end{array}$ & $\begin{array}{l}3 \\
3 \\
\end{array}$ & $\begin{array}{l}39,033 \\
41,994 \\
\end{array}$ & $\begin{array}{l}3 \\
3 \\
\end{array}$ & $\begin{array}{l}33,138 \\
41,580 \\
\end{array}$ & $\begin{array}{l}0.85 \\
0.99 \\
\end{array}$ & $\begin{array}{l}0.94 \\
1.01 \\
\end{array}$ & $\begin{array}{l}0.80 \\
1.00 \\
\end{array}$ \\
\hline \multicolumn{5}{|c|}{ Average $=$} & \multicolumn{2}{|l|}{40,513} & 37,359 & 0.92 & 0.98 & 0.90 \\
\hline $\begin{array}{l}11 \\
11 \\
\end{array}$ & $\begin{array}{l}\mathrm{C} \\
\mathrm{C}\end{array}$ & $\begin{array}{l}19 \\
20 \\
\end{array}$ & $\begin{array}{l}5070-5270 \\
5260-5290 \\
\end{array}$ & $\begin{array}{l}3 \\
3 \\
\end{array}$ & $\begin{array}{l}40,437 \\
40,419 \\
\end{array}$ & $\begin{array}{l}3 \\
3 \\
\end{array}$ & $\begin{array}{l}30,555 \\
36,162 \\
\end{array}$ & $\begin{array}{l}0.76 \\
0.89 \\
\end{array}$ & $\begin{array}{l}0.97 \\
0.97\end{array}$ & $\begin{array}{l}0.74 \\
0.87 \\
\end{array}$ \\
\hline \multicolumn{4}{|c|}{ Average $=$} & \multicolumn{3}{|c|}{40,428} & 33,358 & 0.83 & 0.97 & 0.80 \\
\hline $\begin{array}{l}11 \\
11\end{array}$ & $\begin{array}{l}\mathbf{N} \\
\mathbf{N}\end{array}$ & $\begin{array}{l}19 \\
20\end{array}$ & $\begin{array}{l}5070-5270 \\
5260-5290\end{array}$ & $\begin{array}{l}3 \\
3\end{array}$ & $\begin{array}{l}42,291 \\
44,937\end{array}$ & $\begin{array}{l}3 \\
3\end{array}$ & $\begin{array}{l}32,148 \\
32,625\end{array}$ & $\begin{array}{l}0.76 \\
0.73\end{array}$ & $\begin{array}{l}1.02 \\
1.08\end{array}$ & $\begin{array}{l}0.77 \\
0.79\end{array}$ \\
\hline \multicolumn{5}{|c|}{ Average $=$} & \multicolumn{2}{|l|}{43,614} & 32,386 & 0.74 & 1.05 & 0.78 \\
\hline \multicolumn{5}{|c|}{ Average of all No. 11 bars $^{\S}=$} & 41,518 & & 34,367 & 0.83 & 1.00 & 0.83 \\
\hline \multicolumn{5}{|c|}{ Average of all bars" $=$} & & & & 0.86 & 1.00 & 0.85 \\
\hline
\end{tabular}

*Numerator and denominator based on group average.

${ }^{\dagger}$ Numerator based on group average. Denominator based on average for three deformation patterns for each bar size; each deformation pattern weighted equally.



"Each bar size weighted equally.

$1 \mathrm{psi}=6.89 \mathrm{kPa} ; 1 \mathrm{lb}=4.45 \mathrm{~N}$. 


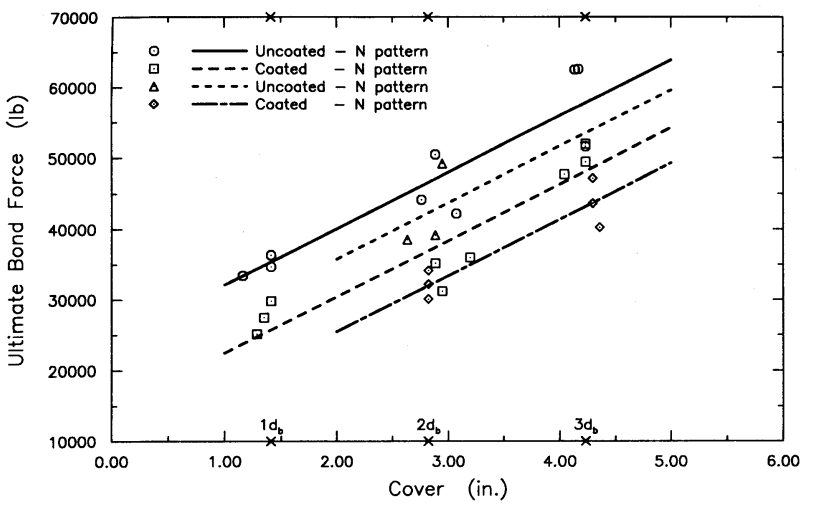

Fig. 6-Ultimate bond force versus cover for No. 11 bars $(1 \mathrm{lb}=4.45 \mathrm{~N} ; 1 \mathrm{in} .=25.4 \mathrm{~mm})$

marized in Table 2. For three out of seven pairs of specimens, the specimens with epoxy-coated reinforcement exhibited a greater maximum crack width than the specimens with uncoated bars. For two pairs, the maximum crack widths were identical, and for two pairs the specimens with uncoated bars had the greater maximum crack width. For four pairs, the specimens with the uncoated bars exhibited a greater number of cracks, while in one pair the two specimens had an identical number of cracks and for two pairs the specimens with the epoxy-coated bars had the greater number of cracks. Thus, at service loads, epoxy-coated bars do not appear to have a significant effect on member behavior, although the data suggests that there may be a slight propensity for increased crack width and crack spacing when coated bars are used.

Table 2 also summarizes the strengths obtained for the splice specimens in terms of bending moment and bar stress. Bar stress is calculated using the usual expression for flexural strength [using equivalent stress block and replacing yield stress with bar stress at ultimate ${ }^{3} \mathrm{cb}$.

Splice specimens with epoxy-coated bars were always weaker than specimens with uncoated bars, with the relative strengths ranging between 0.94 (S-pattern No. 6 bars) and 0.71 (S-pattern No. 11 bars). At failure, all splice specimens exhibited extensive longitudinal and transverse cracking in the region of the splices. Concrete above the splices was easily removed with a hammer, exposing a nearly horizontal crack running the full width of the beam in the plane of the splices.

Bar appearance-The test bars were examined following the tests by removing the concrete cover. Uncoated bars showed evidence of good adhesion to the concrete. Particles of concrete were left on the shaft of the bar and the sides of the deformations. Wedges of compacted concrete powder were lodged in the front of the ribs, adhering to the ribs on the pull side only.

As observed in earlier tests of epoxy-coated reinforcement, ${ }^{1,2}$ there was virtually no evidence of adhesion between the epoxy-coated bars and surrounding concrete. No concrete particles were left on the deformations or the shaft of the coated bars. The concrete in contact with the epoxy-coated bars had a smooth, glassy surface. In a few cases, there were signs of the epoxy coating being crushed against the concrete, but in general the epoxy was undamaged.

\section{EVALUATION OF EXPERIMENTAL RESULTS}

This paper emphasizes the role of bar properties on the bond strength of epoxy-coated reinforcement. Specifically, the roles of coating thickness, deformation pattern, and bar size are studied. In addition, tests designed to validate the test specimen itself are discussed. The ratio of the bond strength of coated bars to the bond strength of uncoated bars, or relative bond strength $C / U$, will be used as the chief measure of the effects of epoxy coating.

Correction factors were applied to beam-end bond strengths to account for variations in cover, coating thickness, and concrete strength. The cover corrections account for deviations in actual concrete cover from the standard of two bar diameters $2 d_{b}{ }^{3}$ This adjustment is obtained by plotting all beam-end specimen strengths for bars of a given size versus the actual cover..$^{10} \mathrm{Cov}$ ers ranging from 1 to $3 d_{b}$ are used (the effect of cover will be addressed in a subsequent paper). The best-fit lines for different groups of specimens are nearly parallel for bars of the same size, independent of deformation pattern or bar surface condition. ${ }^{10}$ Using the technique of dummy variables, ${ }^{11}$ parallel best-fit lines are obtained based on the assumption that changes in cover cause the same incremental change in bond force for bars of the same size, independent of deformation pattern, bar surface condition, and test group. Thus, each group of specimens is represented by a separate line, as shown in Fig. 6 for No. 11 bars. Individual specimen strengths are corrected by shifting the measured bond strength parallel to the best-fit line to a value corresponding to $2 d_{b}$. cover. The impact of this correction is small. An analysis using No. 5 and 6 bar data that was uncorrected for cover altered no conclusions obtained with the cover-corrected data. This is fortunate because a cover correction cannot be made for the No. 8 bars in Groups 2 through 6, since actual cover was not measured for these specimens.

For the epoxy-coated No. 5 bars, a similar correction is necessary based on coating thickness [ 9 mils $(0.23$ $\mathrm{mm}$ ) is taken as the standard], due to the sensitivity of the bond strength of these bars to the thickness of the epoxy. As will be demonstrated, larger bars do not require a coating thickness correction.

In addition to the cover and coating thickness corrections, test results are normalized with respect to a nominal concrete strength of $6000 \mathrm{psi}(41 \mathrm{MPa})$ using the assumption that, within the concrete strength range used, bond strength is proportional to the square root of the compressive strength. Thus, bond strengths are multiplied by $\left(6000 / f_{c}^{\prime}\right)^{1 / 2}$ to obtain the final modified values. Both original and modified values of bond force are summarized in Appendix B and Reference 5. Average modified values of bond force are summarized by bar size, deformation pattern, and group in Table 3 for specimens with standard configurations. 




Fig. 7-Ultimate bond force versus lead length for $\mathrm{N}$ pattern No. 5 bars. Bonded length $=31 / 2$ in. $(1 \mathrm{lb}=$ $4.45 \mathrm{~N} ; 1 \mathrm{in} .=25.4 \mathrm{~mm}$ )

Splice test results are not modified for cover, coating thickness, or concrete strength.

\section{Beam-end specimens}

Specimen evaluation-Due to the large number of variables in the study, it was considered desirable to use a single-bonded length in the beam-end specimens for each bar size. At the outset, however, it was not clear what effect either the bonded length or the lead length had on the reduction in bond strength caused by the epoxy coating. To answer these questions, No. 5 bar specimens with a constant bonded length $3 \frac{1}{2}$ in. ( 89 $\mathrm{mm}$ ) and lead lengths ranging from 0 to $33 / 4$ in. (0 to 95 $\mathrm{mm}$ ), and No. 5, 6, and 8 bar specimens with nonstandard bonded and lead lengths were evaluated. In these latter groups, the nonstandard specimens had longer bonded lengths [No. $5, l_{b}=81 / 2$ in. $(216 \mathrm{~mm})$; No. 6 , $l_{b}=101 / 2$ in. $(267 \mathrm{~mm})$; No. $8, l_{b}=14$ in. $\left.(356 \mathrm{~mm})\right]$ and a shorter lead length $\left[l_{l}=1 / 2\right.$ in. $\left.(13 \mathrm{~mm})\right]$ than the standard test specimens described earlier.

Fig. 7 shows the variation in ultimate bond force as a function of lead length for N-pattern No. 5 bars with a bonded length of $31 / 2$ in. $(89 \mathrm{~mm}$ ) (Groups 7, 8, 11, and 12). As illustrated, the increase in bond strength is nearly linear with increasing lead length for both the coated and uncoated bars. Based on the best-fit lines, $C / U$ varies from only 0.936 to 0.934 for lead lengths of 0 and $33 / 4$ in. ( 0 and $95 \mathrm{~mm}$ ), respectively. Thus, lead length does not appear to play a role in the relative bond strengths of coated and uncoated bars.

Fig. 8 compares the ultimate bond forces of N-pattern No. 5, 6, and 8 bars and S-pattern No. 5 bars as a function of bonded length plus lead length. The data points for the longer total embedment (all from Group 16) represent the average of at least three test specimens. The data points for the shorter embedment represent the average of the standard specimens of each type (corrected to a $2 d_{b}$ cover and No. 5 bars corrected to a nominal 9 mil coating). As illustrated, the ultimate bond force increases with increasing total embedment for No. 5 and 6 bars, but decreases with increasing total embedment for No. 8 bars,. whether the bars are coated or uncoated. This reduction occurs for both

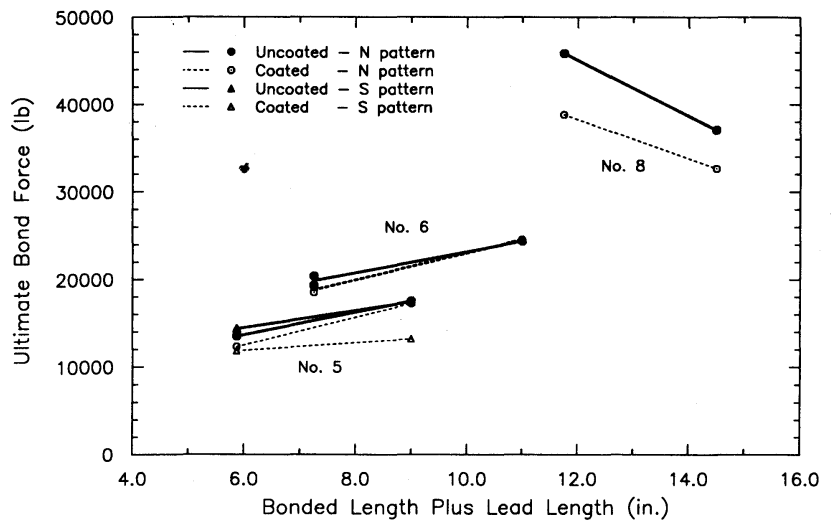

Fig. 8-Ultimate bond force versus bonded length plus lead length for N-pattern No. 5, 6, and 8 bars, and $S$ pattern No. 5 bars $(1 \mathrm{lb}=4.45 \mathrm{~N} ; 1 \mathrm{in} .=25.4 \mathrm{~mm})$

coated and uncoated No. 8 bars. Although not a key aspect of this study, Fig. 7 and 8 show that maximum anchorage capacity does not depend solely on the length of bar in contact with concrete.

Fig. 8 also shows that the bond strengths of coated and uncoated bars respond similarly to changes in specimen geometry, resulting in only small changes in $C / U$. For the N-pattern No. 8 bars, $C / U$ increases from 0.84 for the standard embedment length to 0.88 for the longer embedment length. For the N-pattern No. 6 bars, $C / U$ increases from 0.93 for the standard embedment length to 1.01 for the longer embedment length. For the N-pattern No. 5 bars, $C / U$ increases from 0.91 for the standard embedment length to 0.98 for the longer embedment length, while for the S-pattern No. 5 bars, $C / U$ decreases from 0.83 to 0.76 . When both deformation patterns are considered for No. 5 bars, $C / U$ remains virtually unchanged for the two embedment lengths, with mean values of 0.87 for both standard and longer embedments. Considering the small number of nonstandard specimens tested, none of these variations is statistically significant. Thus, the effect of embedment on $C / U$ remains an open question.

The balance of this report is dedicated to answering the question: Does the effect of the epoxy coating depend on coating thickness, deformation pattern, or bar size? To answer these questions, 20 groups of specimens (Groups 2 through 15 and 17 through 22) were tested. No. 5, 6, and 8 bars were used to evaluate the effect of coating thickness. No. 5, 6, 8, and 11 bars were used to evaluate the effects of deformation pattern and bar size.

Coating thickness-The effect of coating thickness is illustrated in Fig. 9 through 11 for No. 8, 6, and 5 bars, respectively. $C / U$ is plotted as a function of the epoxy coating thickness for each deformation pattern. Each data point represents the ratio of the bond strength of an individual epoxy-coated bar to the average bond strength of uncoated bars with the same deformation pattern and bar size in the same group of specimens. Using the technique of dummy variables, ${ }^{10}$ the best-fit lines for each deformation pattern are obtained using the assumption that there may be differences in the ef- 


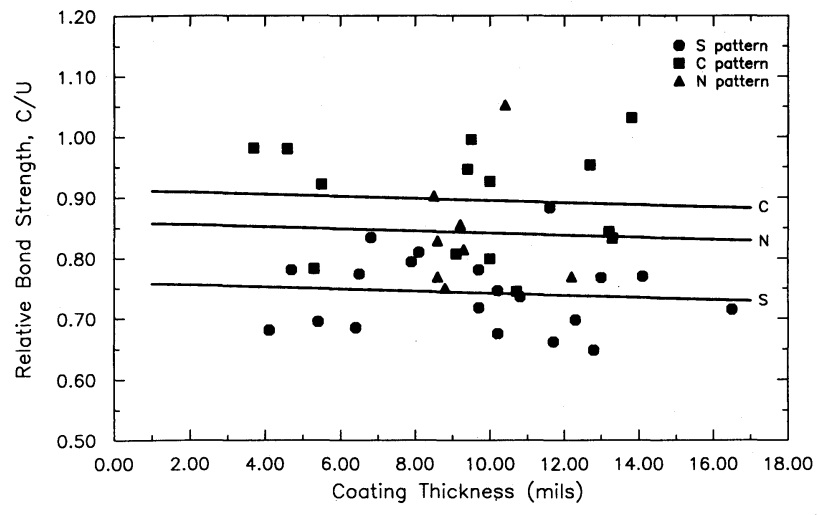

Fig. 9-Relative bond strength $\mathrm{C} / \mathrm{U}$ versus coating thickness for No. 8 bars $(1 \mathrm{mil}=0.0254 \mathrm{~mm})$

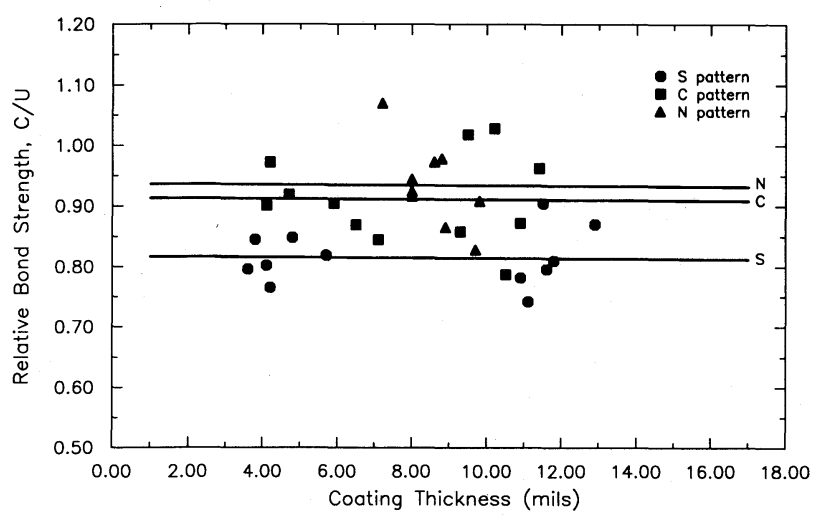

Fig. 10-Relative bond strength $\mathrm{C} / \mathrm{U}$ versus coating thickness for No. 6 bars $(1 \mathrm{mil}=0.0254 \mathrm{~mm})$

fect of the coating due to deformation pattern, but that the effect of coating thickness is the same for all deformation patterns.

Fig. 9 and 10 show that coating thickness plays virtually no role in the magnitude of strength reduction caused by the epoxy coating for No. 8 and 6 bars. This observation matches similar observations made by Johnston and $\mathrm{Zia}^{1}$ and Treece and Jirsa. ${ }^{2}$ The best-fit lines in Fig. 9 and 10 for No. 8 and 6 bars, in fact, have very slight negative slopes, which result in decreases in $C / U$ of 0.012 and 0.002 , respectively, as the coating thickness increases from 5 to 12 mils $(0.13$ to $0.30 \mathrm{~mm})$. In contrast to these observations, Fig. 11 shows that coating thickness does play a role for No. 5 bars, with $C / U$ dropping, on the average, by 0.090 as the coating thickness increases from 5 to 12 mils $(0.13$ to $0.30 \mathrm{~mm})$. This observation does not conflict with earlier studies, ${ }^{1,2}$ since those studies included no bars smaller than No. 6. On reflection, the conclusion that $C / U$ depends on coating thickness for small bars seems completely reasonable because, as bar size decreases, coating thickness becomes more significant in relation to the height of the bar ribs.

Deformation pattern-A second look at Fig. 9 through 11 provides convincing evidence that the effect of the epoxy coating varies considerably with deformation pattern. For the three bar sizes illustrated, the $\mathrm{S}$ pattern is affected the most. The values of $C / U$ for the



Fig. 11-Relative bond strength $\mathrm{C} / \mathrm{U}$ versus coating thickness for No. 5 bars $(1 \mathrm{mil}=0.0254 \mathrm{~mm})$

$\mathrm{C}$ and $\mathrm{N}$ patterns are very close for No. 6 and 5 bars. Also, it can be observed that smaller bars are affected, on the average, less than larger bars. Mean values of $C / U$ based on group, deformation pattern, and bar size are summarized in Table 3 . For a $9 \mathrm{mil}(0.23 \mathrm{~mm})$ coating, the mean values of $C / U$ for the $\mathrm{S}, \mathrm{C}$, and $\mathrm{N}$ deformation patterns are, respectively: $0.83,0.91$, and 0.91 for No. 5 bars; $0.81,0.91$, and 0.93 for No. 6 bars; and $0.74,0.90$, and 0.84 for No. 8 bars.

The results shown in Fig. 9 through 11 do not give a completely equitable comparison of the deformation patterns, because the values of $C / U$ are evaluated individually by deformation pattern. Thus, a coated bar may have a low $C / U$ based on uncoated bars of the same deformation pattern but, in fact, have a higher bond strength than another coated bar that has a high value of $C / U$ because its uncoated bars have a low bond strength. It is fairer to base the values of $C / U$ on the mean strengths of all uncoated bars of the same size. These ratios are listed in Table 3 under the heading " $C / U$, all." Subsequent reference to $C / U$ applies to these values. For a 9 mil coating, the mean values of $C / U$ calculated on this basis for the $\mathrm{S}, \mathrm{C}$, and $\mathrm{N}$ patterns are, respectively: $0.85,0.93$, and 0.87 for No. 5 bars; $0.80,0.89$, and 0.97 for No. 6 bars; $0.73,0.83$, and 0.90 for No. 8 bars; and $0.90,0.80$, and 0.78 for No. 11 bars.

Table 3 also shows the ratios of the mean strengths of uncoated bars in each group to the mean strength of all uncoated bars of the same size $U / U$. The mean values of $U / U$ for the $\mathrm{S}, \mathrm{C}$, and $\mathrm{N}$ patterns are, respectively, 1.03, 1.02, and 0.95 for No. 5 bars; $0.99,0.97$, and 1.04 for No. 6 bars; $0.98,0.96$, and 1.06 for No. 8 bars; and $0.98,0.97$, and 1.05 for No. 11 bars. It is worth noting that not only is the order of relative strength different for coated and uncoated bars of the same size, but the range in the mean values of $C / U$ significantly exceeds the range in the mean values of $U / U$ except for No. 5 bars, where the range of relative strengths is identical. The wider spread in the bond strengths of coated bars emphasizes the strong dependence of bond strength reduction on deformation pattern.

One useful parameter for displaying the results for bars of different sizes is the bearing area ratio $R_{b}{ }^{5,10} R_{b}$ 


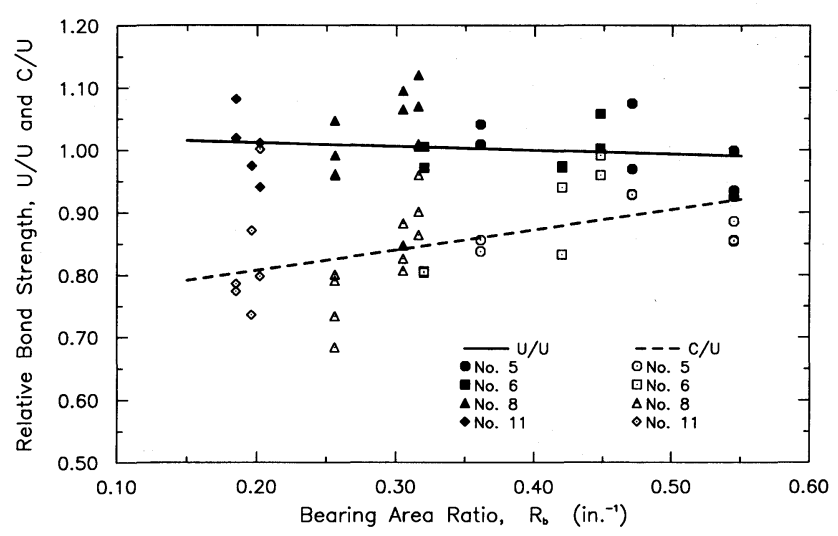

Fig. 12-Relative bond strengths $\mathrm{U} / \mathrm{U}$ and $\mathrm{C} / \mathrm{U}$ versus bearing area ratio $\mathrm{R}_{\mathrm{b}}$ numerator of ratio based on mean bond strength for each group. Denominator based on mean bond strength of all bars of the same size (1 in. $=25.4 \mathrm{~mm}$ )

is equal to the ratio of the rib-bearing area per inch of length to the nominal cross-sectional area of the bar. As illustrated in Table 1 and Fig. 12, $R_{b}$ generally decreases as bar size increases. Also, as shown in Fig. 12, $C / U$ generally decreases as $R_{b}$ decreases. The correlation between $R_{b}$ and $C / U$ cuts across bar size. In contrast, $U / U$ is insensitive to $R_{b}$.

Bar size-The effect of epoxy coating on bond strength as a function of bar size is illustrated in Fig. 13, which compares the relative bond strengths of coated and uncoated bars by deformation pattern. As with Fig. 12, the relative strengths are expressed in terms of the mean strength of uncoated bars of the same size.

For the coated bars, the overall trend is a reduction in $C / U$ with increasing bar size. The mean values of $C / U$ are $0.88,0.89,0.82$, and 0.83 for No. $5,6,8$, and 11 bars, respectively. Based on deformation pattern, the lowest mean values of $C / U$ for each bar size are $0.85,0.80$, and 0.73 , for S-pattern No. 5,6 , and 8 bars, respectively, and 0.78 for N-pattern No. 11 bars.

The $C / U$ values for No. 6 and 11 bars contrast sharply with the mean values obtained by Treece and Jirsa $^{2}$ for splices: 0.74 for No. 6 bars and 0.64 for No. 11 bars.

\section{Splice specimens}

Splice test specimens are larger and more costly than beam-end specimens. Therefore, it is desirable to run fewer splice tests than beam-end tests in a study. There are, however, at least two reasons to run splice tests. Splice tests may provide a more realistic model of what happens in an actual structure, and the development length provisions for epoxy-coated bars in ACI 318-893 are based on the splice tests run by Treece and Jirsa. ${ }^{2}$ With this in mind, it is important to know if beam-end specimens give the same results as splice specimens and if the tests in the current study, both beam-end and splice, match the earlier splice tests. ${ }^{2}$

Before these questions are answered, the variability that is inherent in bond tests should be considered.

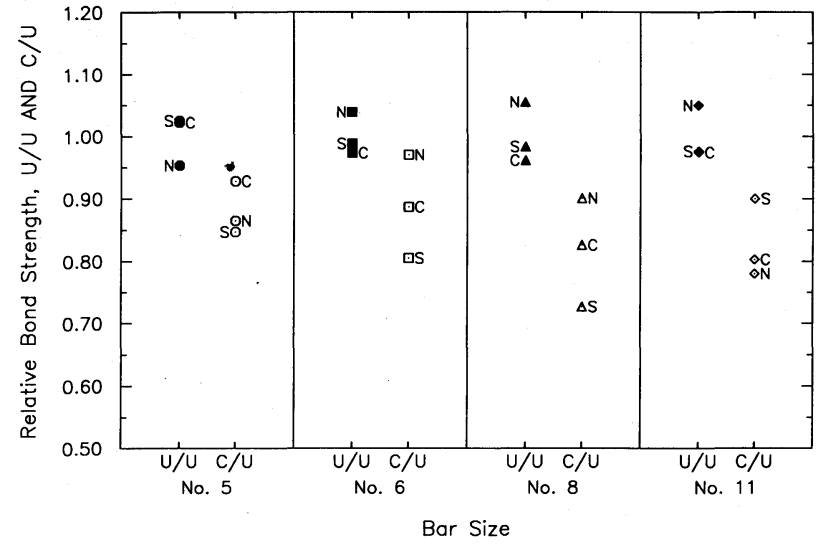

Fig. 13-Relative bond strengths $\mathrm{U} / \mathrm{U}$ and $\mathrm{C} / \mathrm{U}$ versus bar size $(1 \mathrm{in} .=25.4 \mathrm{~mm})$



Fig. 14-Relative bond strength $\mathrm{C} / \mathrm{U}$ versus bearing area ratio $\mathrm{R}_{\mathrm{b}}$. Beam-end and splice specimens from the current study and splice specimens tested by Treece and $\operatorname{Jirsa}^{2}(1 \mathrm{in} .=25.4 \mathrm{~mm})$

Bond tests exhibit a great deal of scatter, as shown in Fig. 9 through 11. However, the scatter shown in these figures is attenuated by the use of the mean values of uncoated bar bond strength in the denominator. If the bond strength of each coated bar is divided by the bond strength of each uncoated bar in the same test group, it is obvious that the scatter in $C / U$ will increase, as shown in Fig. 14. Since the splice tests in this study, as well as those performed by Treece and Jirsa, ${ }^{2}$ were executed with individual coated and uncoated bar specimens, i.e., no replications, the expected scatter in $C / U$ for splices should resemble that shown for the beamend specimens in Fig. 14.

The $C / U$ values for the splice tests in this study and those from Treece and Jirsa ${ }^{2}$ also appear in Fig. 14. As illustrated, the splice tests generally lie within the scatter band but below the mean obtained from the beamend tests. A summary of the splice tests in the current study is presented in Table 2.

For the current study, some splice results are on the high side of the scatter band (S-pattern No. 6, 0.94; S-pattern No. 8, 0.90; and N-pattern No. 8, 0.85) and some are on the low side (N-pattern No. 5, 0.74; C-pattern No. 6, 0.76; and S-pattern No. 11, 0.71). Overall, the plot suggests that the key aspects of bond 
strength reduction caused by epoxy coating are the same for beam-end and splice specimens.

The mean value 0.82 of $C / U$ for the current splice tests is slightly lower than the mean for all beam-end tests, 0.85 . However, the mean value 0.66 of $C / U$ from Treece and Jirsa, ${ }^{2}$ if weighted by test group, or 0.69 if weighted by individual specimen, is considerably below the mean for the beam-end tests. The lower relative strength of the splices can be traced to the fact that most of the splices had a cover less than the $2 d_{b}$ used for the beam-end specimens, and a lower strength is statistically expected for unconfined multiple splice specimens than for single splice or single bar specimens. Detailed consideration of these effects will be included in a future paper.

\section{Implications for design}

The results described here have important implications for design. The major observation is that the bond strength of epoxy-coated bars, relative to uncoated bars, is considerably higher than the value of 0.66 used to calculate the 1.5 development length modification factor for bars with less than $3 d_{b}$ cover in the 1989 ACI Building Code ${ }^{3}$ and 1989 AASHTO Bridge Specifications. ${ }^{4}$ The inverse of the lowest average value of $C / U$ obtained for any bar size or deformation pattern in this study is 1.37 , obtained for S-pattern No. 8 bars. No. 5, 6, and 11 bars are affected even less, with modification factors of $1.18,1.25$, and 1.28 , respectively, based on the deformation pattern with the lowest value of $C / U$. These values are all based on a cover of $2 d_{b}$.

These results suggest that a lower penalty can be employed for coated bars with a $2 d_{b}$ cover than that recommended by Treece and Jirsa ${ }^{2}$ and implemented by $\mathrm{ACI}^{3}$ and $\mathrm{AASHTO}^{4}$ for bars with a cover less than 3 $d_{b}$. It appears that development length modification factors can safely be reduced to 1.25 for No. 6 bars and smaller and 1.35 or 1.40 for No. 7 bars and larger (care should be taken in selecting values for No. 14 and 18 bars, since no tests have been performed on these bar sizes). A modification factor of 1.25 for No. 5 bars and smaller is more than needed, based on a 9 mil coating, but will help to take into account the lower bond strengths obtained by small bars with thicker coatings. Recent work by Cleary and Ramirez* provides additional evidence suggesting that the current design provisions for epoxy-coated bars ${ }^{3,4}$ are overconservative. Before placing these modification factors in a design code, ${ }^{3,4}$ it would be prudent to evaluate at least a portion of the patterns that have not yet been tested.

The results suggest that development length modification factors can be reduced further by altering deformation patterns to improve the bond strength of epoxycoated bars or standardizing on "strong" deformation patterns on an industry-wide basis. Modification factors for each bar size should be based on the deforma- tion pattern with the lowest mean $C / U$ value rather than the mean value of $C / U$ for all bars of a given size, since deformation is clearly a controllable parameter. The deformation pattern tested by Treece and Jirsa ${ }^{2}$ is no longer used for epoxy-coated bars because of difficulties in coating. ${ }^{\dagger}$

The insensitivity to coating thickness of bars larger than No. 5 indicates that coatings thicker than 12 mils $(0.30 \mathrm{~mm})$ could be used on larger bars to improve corrosion protection. This improved protection could be obtained with little reduction in bond strength beyond that currently observed. Additional study is necessary, however, before new limits on coating thickness can be established.

\section{SUMMARY AND CONCLUSIONS}

A large-scale study was carried out to determine the effects of coating thickness, deformation pattern, and bar size on the reduction in bond strength between reinforcing bars and concrete caused by epoxy coating. Beam-end and splice test specimens containing bottomcast No. 5, 6, 8, and 11 bars with average coating thicknesses ranging from 3 to 17 mils $(0.08$ to $0.43 \mathrm{~mm})$ were used. Three deformation patterns were evaluated. All specimens had covers of 2 bar diameters or less.

The following conclusions are based on the results and analyses presented in this paper.

1. Epoxy coatings in the range of 5 to 12 mils $(0.13$ to $0.30 \mathrm{~mm}$ ) significantly reduce the bond strength of deformed reinforcing bars to concrete. However, the extent of the reduction is less than that used to establish the development length modification factors in the 1989 ACI Building Code ${ }^{3}$ and 1989 AASHTO Bridge Specifications. ${ }^{4}$

2. For coatings between 5 and 12 mils $(0.13$ to 0.30 $\mathrm{mm}$ ) in thickness, differences in coating thickness have little effect on the amount of the bond strength reduction for No. 6 bars and larger. Thicker coatings cause a greater reduction in bond strength than thinner coatings for No. 5 bars.

3. In general, the reduction in bond strength caused by epoxy coating increases with bar size.

4. The magnitude of the reduction depends on deformation pattern. Bars with relatively larger rib-bearing areas with respect to the bar cross section are affected less by the coating than bars with smaller bearing areas.

\section{ACKNOWLEDGMENTS}

Major funding for this research was provided by the National Science Foundation under Grant No. ECE-8616228. Reinforcing steel was supplied by Chaparral Steel Company, North Star Steel Company, Sheffield Steel Corporation, and Structural Metals, Inc. The epoxy coating was applied by ABC Coating Company, Inc. and Simcote, Inc. Additional support was provided by the University of Kansas Transportation Center.
*Cleary, Douglas B., and Ramirez, Julio A.; “'Bond Strength of Epoxy
Coated Reinforcement," $A C I$ Materials Journal, in press.
${ }^{\dagger}$ Florida Steel Corporation, personal communication. 


\section{REFERENCES}

1. Johnston, David W., and Zia, Paul, "Bond Characteristics of Epoxy Coated Reinforcing Bars," Report No. FHWA-NC-82-002, Federal Highway Administration, Washington, DC, 1982, 163 pp.

2. Treece, Robert A., and Jirsa, James O., "Bond Strength of Epoxy-Coated Reinforcing Bars," ACI Materials Journal, V. 86, Nó. 2, Mar.-Apr. 1989, pp. 167-174.

3. ACI Committee 318, "Building Code Requirements for Reinforced Concrete (ACI 318-89) and Commentary (ACI 318R-89)," American Concrete Institute, Detroit, 1989, $353 \mathrm{pp.}$

4. Standard Specification for Highway Bridges, 14th ed., American Association of State Highway and Transportation Officials, Washington, D.C., 1989, 420 pp.

5. Choi, Oan Chul; Hadje-Ghaffari, Hossain; Darwin, David; and McCabe, Steven L., "Bond of Epoxy-Coated Reinforcement to Concrete: Bar Parameters," SL Report 90-1, University of Kansas Center for Research, Lawrence, Jan. 1990, 43 pp.

6. Brettmann, Barrie B.; Darwin, David; and Donahey, Rex C.,
"Bond of Reinforcement to Superplasticized Concrete," ACI JourNAL, Proceedings V. 83, No. 1, Jan.-Feb. 1986, pp. 98-107.

7. "Standard Specification for Deformed and Plain Billet-Steel Bars for Concrete Reinforcement," (ASTM A 615-87a) 1989 Annual Book for ASTM Standards, V. 1.04, ASTM, Philadelphia, pp. 381384.

8. "Standard Specification for Epoxy-Coated Reinforcing Steel Bars," (ASTM A 775/A775M-88a) 1989 Annual Book for ASTM Standards, V. 1.04, ASTM, Philadelphia, pp. 548-552.

9. Donahey, Rex C., and Darwin, David, "Bond of Top-Cast Bars in Bridge Decks," ACI Journal, Proceedings V. 82, No. 1, Jan.Feb. 1985, pp. 57-66.

10. Choi, O. C.; Darwin, D.; and McCabe, S. L., "Bond Strength of Epoxy-Coated Reinforcement to Concrete," SM Report No. 25, University of Kansas Center for Research, Lawrence, July 1990, 217 pp.

11. Draper, N. R., and Smith, H., Applied Regression Analysis, 2nd ed., John Wiley \& Sons, New York, 1981, pp. 241-249. 